\title{
The Relation Between the Alternating Group and Standard Row Young's Diagrams
} \section{Department of Mathematics, College of Education, University of Al-Hamdaniya, Mosul, Iraq .}

Ilham Matta Yacoob, Hadil H. Sami, Mohammed Kassim Ahmed

Received $26 / 12 / 2017$
Department of Computer Science, College of Education, University of Al-Hamdaniya, Mosul, Iraq

\section{Accepted}

23 / 01 / 2018

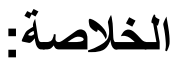

في هذا البحث قدمنا العلاقة بين الزمرة المتتاوبة A ومخططات يونك في حالة الصفوف القياسية، فقد تم تقسيم العمل الى مرحلتين ... المرحلة الاولى هو ايجاد عدد التبديلات الزوجية بالاعتماد على مفهوم التجزئة ب من خلال انشاء خوارزمية لهذا الغرض، والمرحلة الثانية من خلال ايجاد علاقة تربط بين طول الدورة والتجزئة بر.
\end{abstract}

\section{Abstract:}

This study tackles the relationship between the alternating group $A_{n}$ and young's diagrams concerning standard rows. The study has been divided into two stages... First, even permutations have been found depending on the conception of partition $\mu$ through formulating an algorithm for this purpose. Secondly, the relationship between the cycle length and partition $\mu$ has been found.

\subsection{Introduction:}

Let $n$ be a non-negative integer, a composition $\mu$ of $n$ is a sequence $\mu=\left(\mu_{1}, \mu_{2}, \cdots \mu_{\mathrm{r}}\right)$ of non-negative integers such that $|\mu|=\sum_{j=1}^{r} \mu_{j}=n$, [5]. For example, if $n=4$, the following sequences are compositions:

(4), (3,1), (2,2), (1,3), (2,1,1), (1,2,1), (1,1,2), (1,1,1,1). 
A composition $\mu$ is said to be a partition for $n$ if $\mu_{j} \geq \mu_{j+1}$, [5]. In this case of the above mentioned example $n=4$, the following sequences realize the condition of partition: $(4),(3,1),(2,2),(2,1,1),(1,1,1,1)$. young's diagrams [5] for partition $\mu=\left(\mu_{1}, \mu_{2}, \cdots \mu_{\mathrm{r}}\right)$ of $n$ is :

$$
[\mu]=\left\{(x, y): 1 \leq y \leq \mu_{\mathrm{x}}, x \geq 1\right\} \subseteq \mathrm{N} \times \mathrm{N}
$$

The elements of $[\mu]$ are called nodes for partition $\mu$ of $n$, and these nodes are elements from $\mathrm{N} \times \mathrm{N}$, it is represented by a diagram in the form of a system of adjacent square boxes, where $\mu_{1}$ of squares are included in the upper row followed by $\mu_{2}$ boxes in the row that follows and so on. for example, young diagrams for partition $\mu$ in case of $n=2$ is

$$
\begin{array}{ll}
\mu_{1}=(2)=\square \square, & \mu_{2}=(1,1)=\square \\
\text { In case of } n=3: & \square \\
\mu_{1}=(3)=\square \square \square & , \quad \mu_{2}=(2,1)=\square \quad, \mu_{3}=(1,1,1)=\square
\end{array}
$$

It is said that the rows of young diagram are standard if the numbers 1 to $n$ are included in each row increasingly [7]. For example, the permutations realized in the case of partition $(2,1)$ are only:

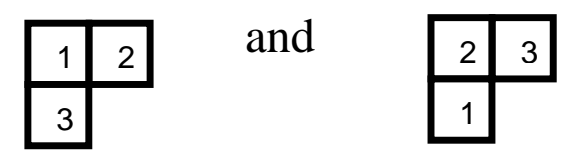

[1] If $f \in S_{n}$, then $f$ is said to be an even (odd) permutation if and only if the multiplication output is:

$$
\prod_{i>k} \frac{f(i)-f(k)}{i-k}=\left\{\begin{array}{c}
1 \text { then } f \text { is even } \\
-1 \text { then } f \text { is odd }
\end{array} \quad \forall i, k=1,2,3, \ldots, n\right.
$$

For example, the permutation $f=\left(\begin{array}{llll}1 & 2 & 3 & 4 \\ 2 & 3 & 1 & 4\end{array}\right) \in S_{n}$ is even because:

$$
\begin{aligned}
& \prod_{i>k} \frac{f(i)-f(k)}{i-k}= \\
& \left(\frac{f(4)-f(3)}{4-3}\right)\left(\frac{f(4)-f(2)}{4-2}\right)\left(\frac{f(4)-f(1)}{4-1}\right)\left(\frac{f(3)-f(2)}{3-2}\right)\left(\frac{f(3)-f(1)}{3-1}\right) \\
& \left(\frac{f(2)-f(1)}{2-1}\right)=1
\end{aligned}
$$




\section{Ilham Matta Yacoob, Hadil H. Sami \& Mohammed Kassim Ahmed}

The alternating group $A_{n}$ is defined as the group of all even permutations on a finite group, and it is a subgroup of a symmetric group $S_{n}$ with $\frac{\mathrm{n} !}{2}$ elements. [6]

The following algorithm has been formulated to find the even cases in $S_{n}$ *

Algorithm (1.1)
Begin
$\mathrm{s} \leftarrow$ size of group
$\mathrm{h} \leftarrow 1$
$\mathrm{~N} \leftarrow$ factorial (s)
Prod $\leftarrow 1$
Repeat for $\mathrm{x} 1=[1$ to $\mathrm{s}]$

$\{\quad$ Repeat for $\mathrm{x} 2=[1$ to $\mathrm{s}]$

$\mathrm{a}=\left[\begin{array}{ll}\mathrm{x} 1 & \mathrm{x} 2\end{array}\right]$

if (a has no equal elements)

$\{$ Repeat for $\mathrm{x} 3=[1$ to $\mathrm{s}]$

$\mathrm{a}=\left[\begin{array}{lll}\mathrm{x} 1 & \mathrm{x} 2 & \mathrm{x} 3\end{array}\right]$

if (a has no equal elements)

$\{$ Repeat for $\mathrm{x} 4=[1$ to s]

$\mathrm{a}=\left[\begin{array}{llll}\mathrm{x} 1 & \mathrm{x} 2 & \mathrm{x} 3 & \mathrm{x} 4\end{array}\right]$

if (a has no equal elements)

$\{$ Repeat for $\mathrm{x} 5=[1$ to s]

$\left\{\mathrm{a}=\left[\begin{array}{lllll}\mathrm{x} 1 & \mathrm{x} 2 & \mathrm{x} 3 & \mathrm{x} 4 & \mathrm{x} 5\end{array}\right]\right.$

if (a has no equal elements)

\{

Repeat until: $\quad \mathrm{a}=\left[\begin{array}{llll}\mathrm{x} 1 & \mathrm{x} 2 & \ldots & \mathrm{xs}\end{array}\right]$

if (a has no equal elements)

$\{$ Prod $\leftarrow 1$

Repeat for $\mathrm{c}=\mathrm{s}$ to 1 steps $(-1)$

$\{\quad$ Repeat for $\mathrm{d}=\mathrm{c}-1$ to 1 steps $(-1)$

$\left\{\right.$ prod $\leftarrow$ prod $* \frac{a(c)-a(d)}{c-d}$

\}

if (prod $>=0$ )

add a to alt matrix at row $h$

$\mathrm{h} \leftarrow \mathrm{h}+1$

\} 


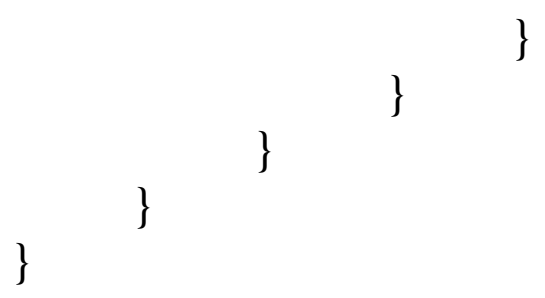

End

[2] The permutations that can be written as a cycle of length 2. Such cycles are called transpositions, that is:

$\left(a_{1}, a_{2}, \cdots, a_{n}\right)=\left(a_{1} a_{n}\right)\left(a_{1} a_{n-1}\right) \cdots\left(a_{1} a_{3}\right)\left(a_{1} a_{2}\right)$.

[2] A permutation of a finite set is even or odd according to weather it can be expressed as a product of an even number of transposition or the product of an odd number of transpositions.

The following facts can be used:

1) The product of two of even or odd permutations will be even permutation.

2) The product of odd and even permutation will be odd.(similarly if it is even permutation and odd permutation, it will be odd).

Example: permutations $f_{1}=(15342), f_{2}=(23)(45) \in S_{5}$ are even and belong to $A_{n}$ because:

$f_{1}=(15342)$

$=(12)(14)(13)(15)$

Since the number of transpositions is even, therefore $f_{1}$ permutation is even. $f_{2}=(23)(45)$

It is obvious it is even permutation, it is also possible to benefit from the facts stated in the previous definition.

Note: the identity element is considered even because it could be written as the product of $\mathrm{p}$ transpositions, i.e $i d=\tau_{1} \tau_{2 \ldots} \tau_{p}$ where $\mathrm{p}$ is even.

In this way it is possible to find all the even permutations in the alternating group $A_{n}$. For example in

$$
\begin{aligned}
& A_{2}=\frac{2 !}{2}=1 \\
& =\{(1)(2)\}
\end{aligned}
$$

That is, the identity element only

$$
\begin{aligned}
& A_{3}=\frac{3 !}{2}=3 \\
& =\{(1)(2)(3),(123),(132)\}
\end{aligned}
$$




$$
\begin{aligned}
& A_{4}=\frac{4 !}{2}=12 \\
& =\left\{\begin{array}{c}
(1)(2)(3)(4),(1)(234),(1)(243),(12)(34),(123)(4),(124)(3), \\
(132)(4),(134)(2),(13)(24),(142)(3),(143)(2),(14)(23)
\end{array}\right\} .
\end{aligned}
$$

\section{2 . 2 The Alternating Group and Young Diagram}

In this section, we detected the number of even permutations from $\mathrm{A}_{\mathrm{n}}$ which corresponds the partition $\mu$ to $n$ relate to young diagrams in the case of standard rows through an algorithm for counting the number as well as showing all the cases for each partition. Here, two cases appear: the first is called general and the second will be tackled in specific cases as illustrated below:
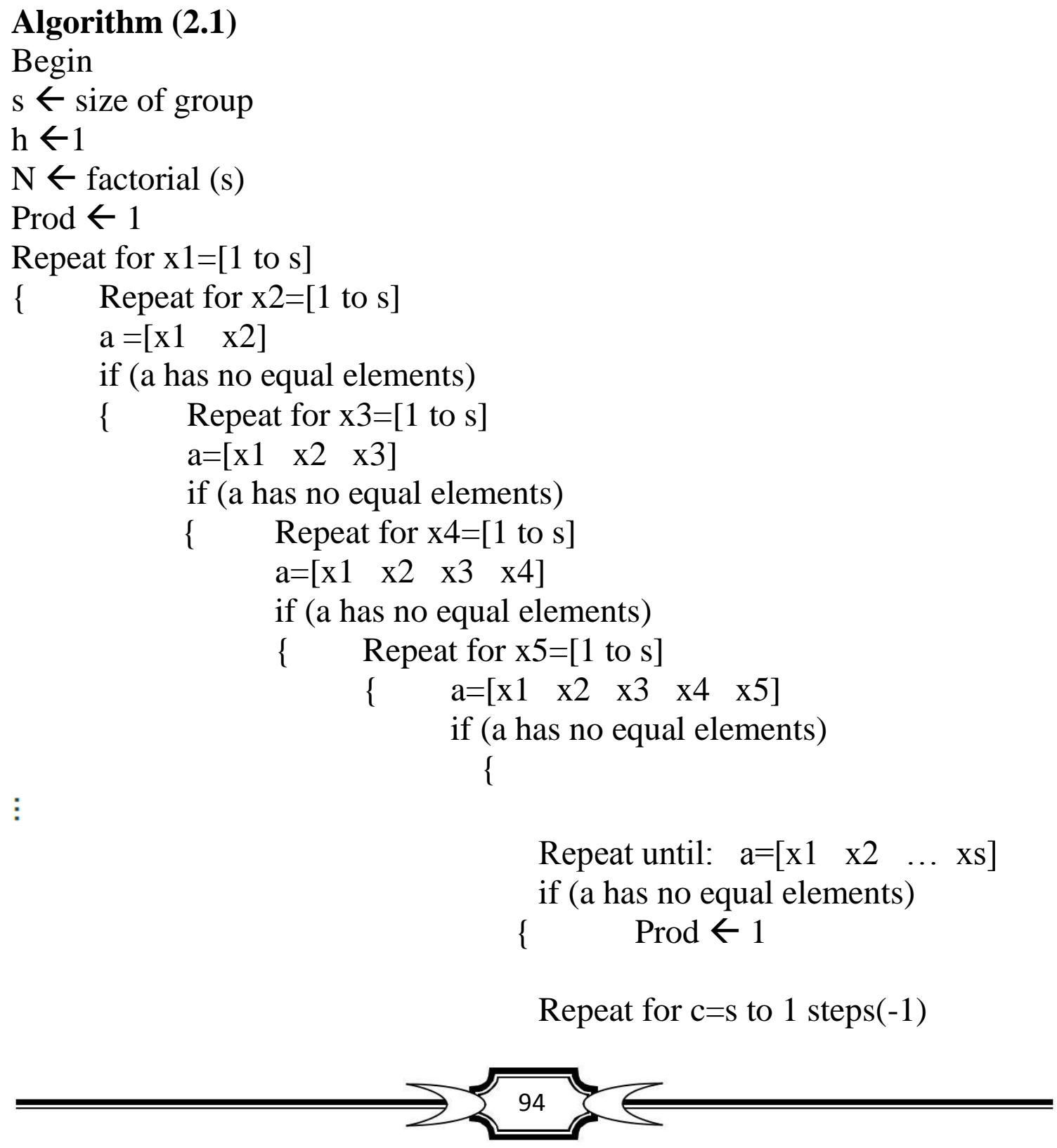


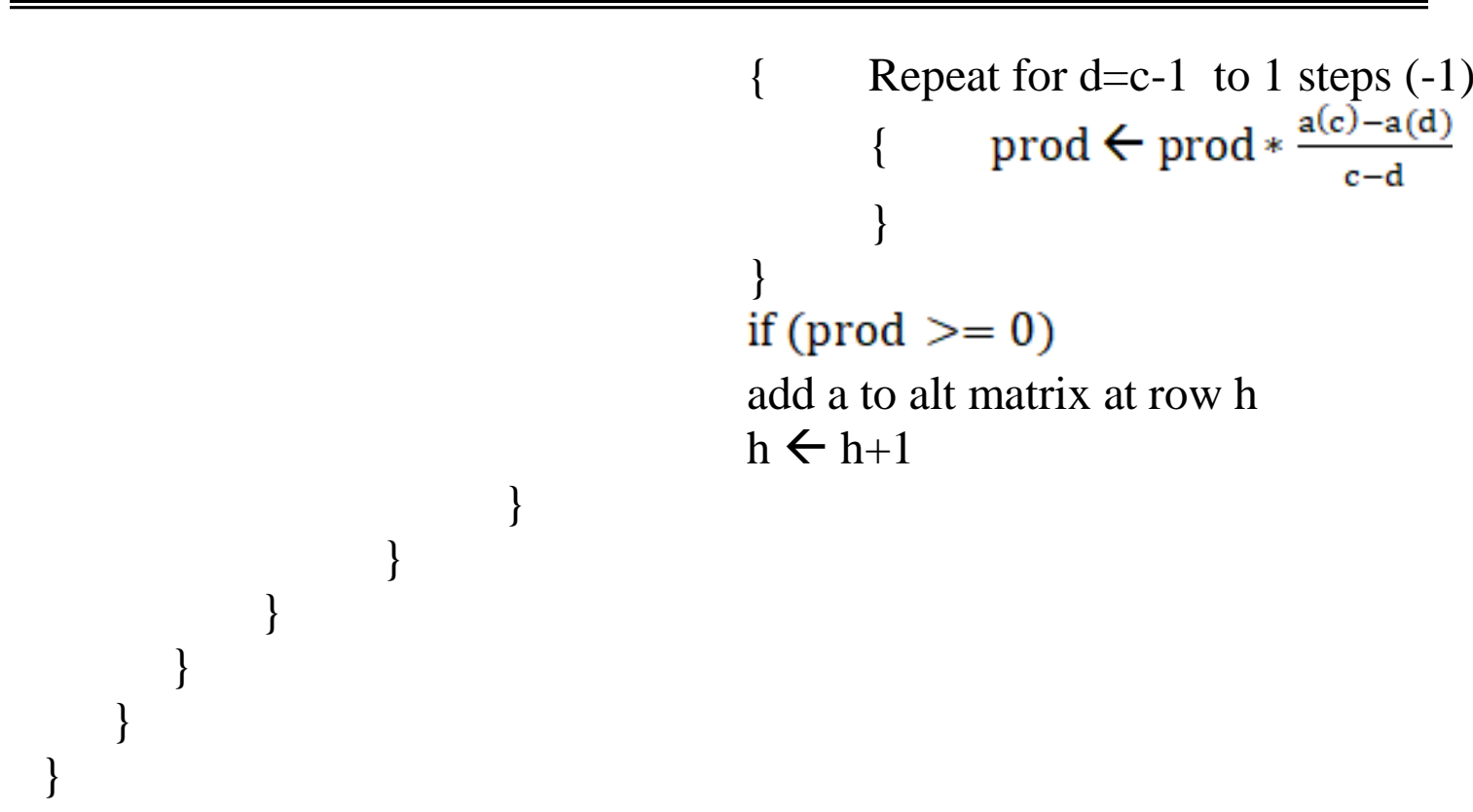

// Set initial variable values:

$\mathrm{AS} \leftarrow 0, \mathrm{~A}(\mathrm{~S}-1)(1) \leftarrow 0, \mathrm{~A}(\mathrm{~S}-2)(2) \leftarrow 0, \mathrm{~A}(\mathrm{~S}-2)(1)(1) \leftarrow 0 \ldots \mathrm{A}(1)(1) \ldots$ $(1) \leftarrow 0$.

Repeat for $\left[\mathrm{i}=1\right.$ to $\left.\frac{\text { factorial }(s)}{2}\right]$

\{

// Checking if rows that have values (1 to s) are sorted

if Alt (row i) is sorted add Alt (row i) to ArS matrix at row AS

$\mathrm{AS} \leftarrow \mathrm{AS}+1$

// Checking if rows that have values (1 to s-1) are sorted

if Alt (row I, columns 1 to s-1) is sorted

add Alt (row i) to $\operatorname{Ar}(\mathrm{S}-1)(1)$ matrix at row $\mathrm{A}(\mathrm{S}-1)(1)$

$\mathrm{A}(\mathrm{S}-1)(1) \leftarrow \mathrm{A}(\mathrm{S}-1)(1)$

// Checking if both rows that have values (1 to s-2) and (s-1 to s) are sorted if Alt (row I, columns 1 to s-2) is sorted and Alt (row I, columns s-1 to s) is sorted

add Alt (row i) to $\operatorname{Ar}(\mathrm{S}-2)(2)$ matrix at row $\mathrm{A}(\mathrm{S}-2)(2)$

$\mathrm{A}(\mathrm{S}-2)(2) \leftarrow \mathrm{A}(\mathrm{S}-2)(2)+1$

\}

End

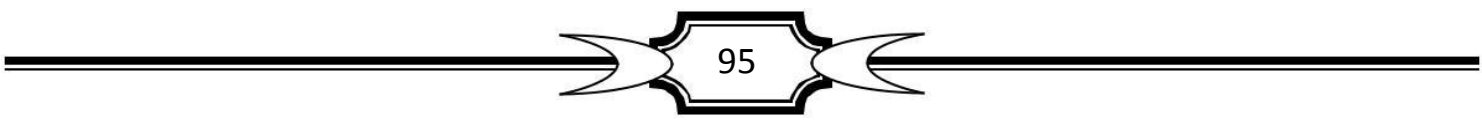




\section{Ilham Matta Yacoob, Hadil H. Sami \& Mohammed Kassim Ahmed}

Proposition (2.2): the number of even permutations $\sigma(\mu)$ in $A_{n}$ for any partitioning $\mu=\left(\mu_{1}, \mu_{2}, \cdots \mu_{\mathrm{r}}\right)$ is:

$\sigma(\mu)=\frac{n !}{2\left(\mu_{1} ! \mu_{2} ! \cdots \mu_{r} !\right)}$

Proof: According to [7], the law in a symmetric group is:

$\mathrm{M}^{\mu}=\frac{\mathrm{n} !}{\mu_{1} ! \mu_{2} ! \cdots \mu_{\mathrm{r}} !}$

Since the relationship between the symmetric and the alternating is $\frac{s_{n}}{2}$, therefore

$\sigma(\mu)=\frac{n !}{2\left(\mu_{1} ! \mu_{2} ! \cdots \mu_{r} !\right)}$

\section{Proposition (2.3) exceptional cases}

Building on using the general rule (2.2) and comparing the results that appeared with the computer program, certain exceptional cases are detected that rule (2.2) cannot be applied on it, because if it is used, it will not yield accurate results that result in correct solution and due to some reasons like the possibilities of computer and an electricity system in the country which does not allow the program to continue to work until the end without turn off. Hence we worked until $A_{7}$, so we could not find a suitable base to work on it. Therefore, we presented them as exceptional cases as follows:

(2.3.1) if $\mathrm{n}$ is odd; we will have the following cases:

a) $(n-1,1)$

b) $\left(2^{q}, 1\right)$ where $q=1,2, \ldots$

c) $(n-3,3)$

d) $(4,3, l)$ where $l=0,1,2, \ldots$

e) $\left(z, 2^{q}, 1\right) \quad$ where $z=2 l, \quad l \geq 0, \mathrm{q}=1,2, \ldots$

(2.3.2) if $\mathrm{n}$ is even; we will have the following case:

$\left(2^{q}\right) \quad$ where $q=1,2, \ldots$

(2.3.3) if $n$ is even or odd, the following will be the case:

$$
(n-2,2)
$$

To illustrate this, we will take the cases $A_{2}$ to $A_{4}$, all other cases can be found in the computer program. $A_{n}^{*}$ is used to indicate the realization of

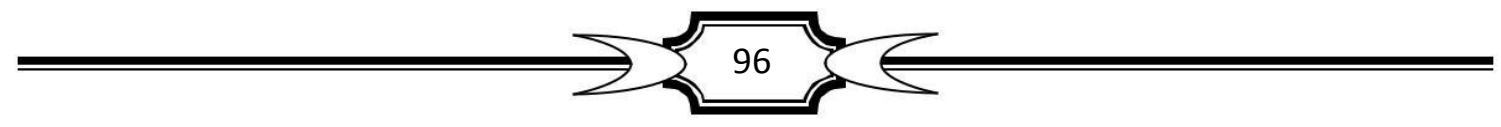


the alternating group conditions and the standard rows of young diagram as well.
$A_{2}^{*}=$
$\left\{(2)=1 \longrightarrow \begin{array}{|l|l|}\hline 1 & 2 \\ \hline\end{array}\right.$
and $\quad(1,1)=\$ \quad 1$
$\mathrm{A}_{3}^{*}=$
$\left\{(3)=1 \longrightarrow \begin{array}{|l|l|l}\hline 1 & 2 & 3 \\ \hline\end{array} \quad\right.$ (2,1)=2 $\rightarrow \begin{array}{|l|l|}\hline 2 & 3 \\ \hline 1 & \end{array}$ and

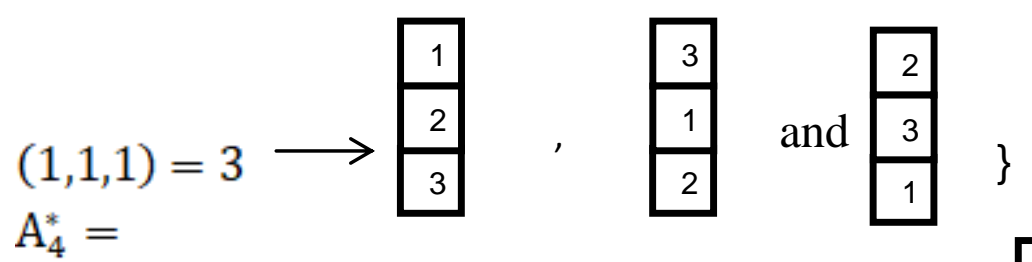

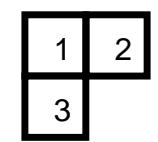

$\left\{(4)=1 \rightarrow \begin{array}{|l|l|l|l|}\hline 1 & 2 & 3 & 4 \\ \hline\end{array}\right.$

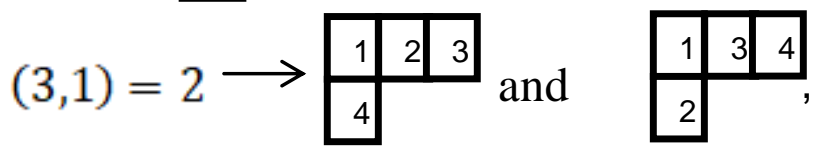

$(2,2)=4$

\begin{tabular}{|l|l|}
\hline 1 & 2 \\
\hline 3 & 4 \\
\hline
\end{tabular}, \begin{tabular}{|l|l|}
\hline 3 & 4 \\
\hline 1 & 2 \\
\hline
\end{tabular}, \begin{tabular}{|l|l|}
\hline 1 & 4 \\
\hline 2 & 3 \\
\hline
\end{tabular}

and

\begin{tabular}{|l|l|}
\hline 2 & 3 \\
\hline 1 & 4 \\
\hline
\end{tabular}

$(2,1,1)=6$

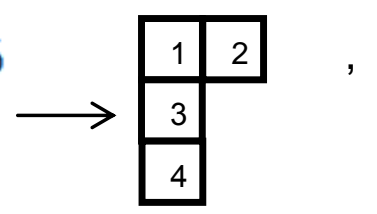

\begin{tabular}{|l|l|}
\hline 3 & 4 \\
\hline 1 & \\
\cline { 1 - 1 } 2 &
\end{tabular}

\begin{tabular}{|l|l|}
\hline 1 & 3 \\
\hline 4 & \\
\cline { 1 - 1 } 2 & \\
\cline { 1 - 1 }
\end{tabular}

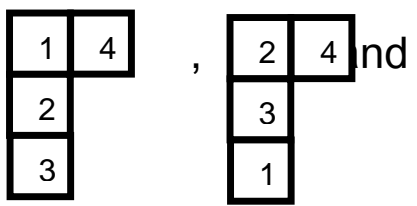

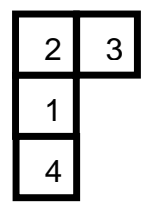

$(1,1,1,1)=12 \longrightarrow$
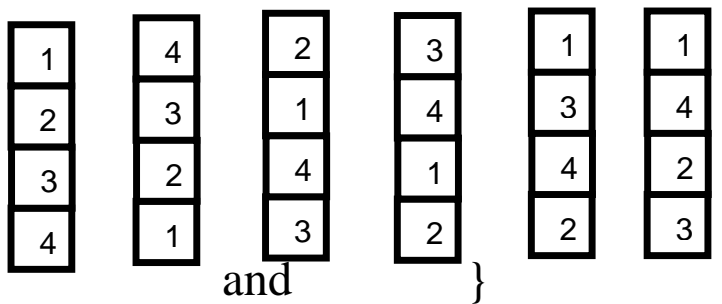

\begin{tabular}{|l|}
\hline 3 \\
\hline 1 \\
\hline 2 \\
\hline 4 \\
\hline
\end{tabular}

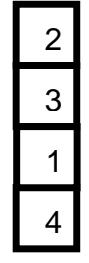

\begin{tabular}{|l|}
\hline 4 \\
\hline 1 \\
\hline 3 \\
\hline 2 \\
\hline
\end{tabular}
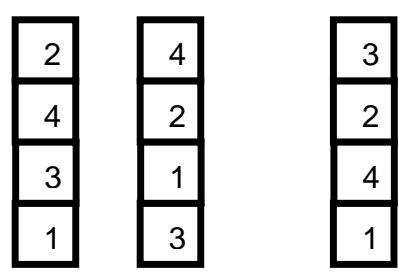

To count the number of even permutations in the partition $(3,1)$ and $(2,2)$ concerning the alternating group $A_{4}$ by using $(2.1)$ and $(2.2)$, the permutation will be:

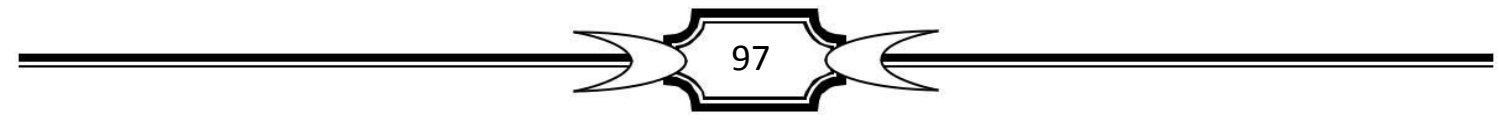




$$
\begin{aligned}
\sigma(3,1) & =\frac{4 !}{2(3 ! 1 !)} \\
& =2 \\
\sigma(2,2) & =\frac{4 !}{2(2 ! 2 !)} \\
& =3
\end{aligned}
$$

As illustrated $\sigma(2,2)$ is considered one of the specific cases according to (2.3).

\subsection{Compute the Even Permutations by Using the Length of \\ Cycles and Partition}

First, we will refer to the concept of conjugation classes in general. The row of conjugate is defined as follows, if we have the two elements $\mathrm{x}, \mathrm{y}$ in a group $\mathrm{G}$, then $\mathrm{x}=g^{-1} \mathrm{y} g$ for each $g \in \mathrm{G}$ is an equivalence relationship on $\mathrm{G}$, hence the rows of equivalence are called rows of conjugates for $\mathrm{G}$ [3]. It's a fact that each two elements in $S_{n}$ are conjugates if they have the same proposition or length of the cycle as $A_{n}$. The construct cycle represents a series of non- negative integers symbolized $\left\{u_{r}\right\}$ where $r=1,2, \ldots, i, u_{r}$ represents all the non- interlinked symmetric cycles, where $\sum_{r=1}^{i} u_{r}=n,[4]$. The length of the cycle can be defined as follows, if we have $\in S_{n}$, we say $f$ is a cycle and its length (K-cycle), $f=\left(n_{1}, n_{2}, \cdots, n_{K}\right)$ if $f\left(n_{i}\right)=n_{i+1}$ for $1 \leq i<\mathrm{K}$ and $f\left(n_{K}\right)=n_{1}$, also $f(n)=n$ when $n \neq n_{1}, n_{2}, \cdots, n_{K} \cdot[1]$

As an illustration, we will provide all the lengths of the cycles in $\mathrm{A}_{2}$ to $\mathrm{A}_{4}$, and later we will provide tables showing the lengths and number of young diagrams that appropriate each length until $\mathrm{A}_{7}$.

$$
\begin{aligned}
& \mathrm{A}_{2}=\{(1)(2)\} \longrightarrow \mathrm{K}\left(\mathrm{A}_{2}\right)=1^{2} \\
& \mathrm{~A}_{3}=\{(1)(2)(3),(123),(132)\} \longrightarrow \mathrm{K}\left(\mathrm{A}_{3}\right)=\left\{1^{3}, 3,3\right\}
\end{aligned}
$$

This means we have only two types of conjugate rows in which the permutations are even and they are 3- cycle and 1-cycle

$$
\begin{aligned}
& \mathrm{A}_{4}=\left\{\begin{array}{c}
(1)(2)(3)(4),(1)(234),(1)(243),(123)(4),(124)(3),(132)(4), \\
(134)(2),(142)(3),(143)(2),(13)(24),(12)(34),(14)(23)
\end{array}\right\} \longrightarrow \\
& \mathrm{K}\left(\mathrm{A}_{4}\right)=\left\{1^{4}, 13,13,13,13,13,13,13,13,2^{2}\right\}
\end{aligned}
$$


Here in the case of $\mathrm{A}_{4}$ we have three types of conjugate rows in which the permutations are even and they are $(3-$ cycle $)(1-$ cycle $),(1-$ cycle $)$ and$$
(2-\text { cycle })(2-\text { cycle }) \text {. }
$$

Now we will offer some rules that account for the number of even permutations in $\mathrm{A}_{n}$.

Rule (3.1): If $n$ is odd, the cycle length $n=(r)$, however if $n$ is even, the cycle length is $n=(1)(r-1)$ for any partition $\mu=\left(\mu_{1}, \mu_{2}, \cdots \mu_{r}\right)$, the number of even permutations that are symbolized $\beta_{1}$ will be:

$$
\beta_{1}= \begin{cases}\frac{n !}{(r-1) ! \mu_{1} ! \mu_{2} ! \cdots \mu_{r} !} & \text { if } n \text { is even } \\ \frac{n !}{(r) ! \mu_{1} ! \mu_{2} ! \cdots \mu_{r} !} & \text { if } n \text { is odd }\end{cases}
$$

This use is illustrated in the following tables, the last column in each table.

Rule (3.2): In the case of partition $\left(1,1, \ldots, 1_{r}\right)$ and all case of length indicated in white color in the last row of each table in the following tables, the relationship will be symbolized $\beta_{2}$ as pointed out by [4] as in:

$\beta_{2}=\frac{n !}{\prod_{r} r^{u_{r}} u_{r} !}$

Rule (3.3): Here some exceptional cases appeared as it is the case in the second section that will displayed in details. This case has been treated by using even permutations in $\mathrm{A}_{2}$ till $\mathrm{A}_{7}$ as follows:

(3.3.1) if $\mu=(n)$, the standard rows illustrated in the following tables in yellow will be according to the length of the cycle as follows:

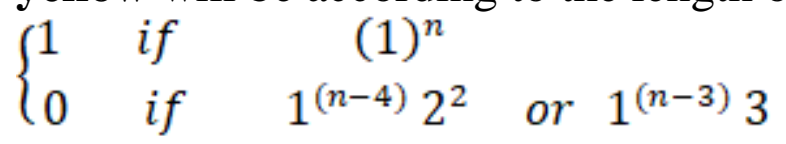

(3.3.2) if $\mu=(r, 1)$, the standard rows in light orange in the following tables will be according to the cycle length as follows:
$\begin{cases}1 & \text { if } \\ 0 & \text { if }\end{cases}$
$(1)^{n}$ or
$1^{(r-2)} 3$ $1^{(r-3)} 2^{2}$ 
(3.3.3) if $\mu=(r, 2)$, the standard rows in light blue in the following tables will be according to the cycle length as follows:

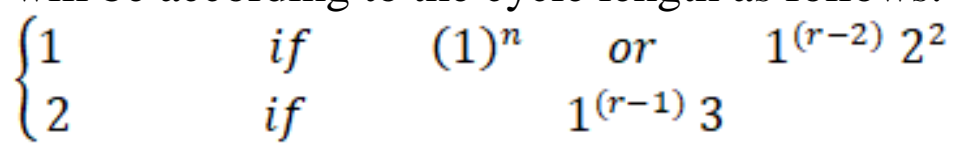

(3.3.4) if $\mu=(r, 3)$, the standard rows in dark green in the following tables will be according to the cycle length as follows:

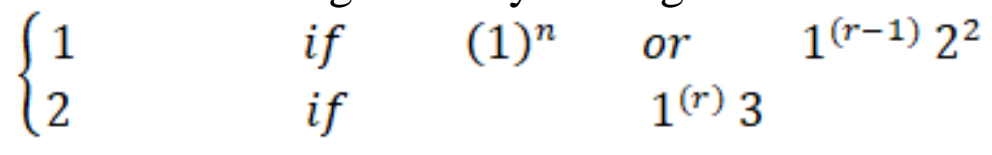

(3.3.5) if $\mu=(r, 1,1)$, the standard rows in light green in the following tables will be according to the cycle length as follows:
$\left\{\begin{array}{l}1 \\ 4\end{array}\right.$
if
$(1)^{n}$
or $1^{(r-2)} 2^{2}$
$1^{(r-1)} 3$

(3.3.6)
tables
3
6 tables will be according to the cycle length as follows:

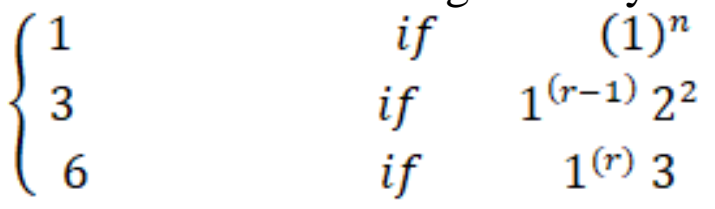

(3.3.7) if $\mu=(r, 3,1)$, the standard rows in light brown in the following tables will be according to the cycle length as follows:
$\left\{\begin{array}{l}1 \\ 3 \\ 6\end{array}\right.$
if $(1)^{n}$
if $\quad 1^{(r)} 2^{2}$
if $\quad 1^{(r+1)} 3$

(3.3.8) if $\mu=(r, 1,1,1)$, the standard rows in dark orange in the following tables will be according to the cycle length as follows:
$\left\{\begin{array}{c}1 \\ 6 \\ 11\end{array}\right.$
if
$(1)^{n}$
if $\quad 1^{(r-1)} 2^{2}$
if
$1^{(r)} 3^{2}$

(3.3.9) if $\mu=(r, 2,2)$, the standard rows in dark blue in the following tables will be according to the cycle length as follows:

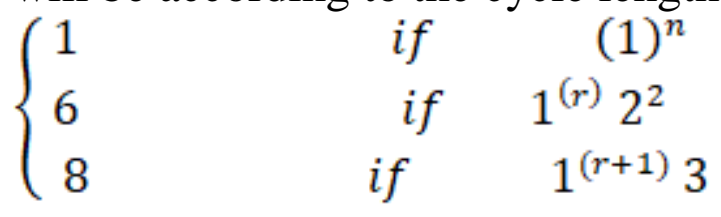

(3.3.10) if $\mu=(r, 2,1,1)$, the standard rows in dark purple in the following tables will be according to the cycle length as follows: 


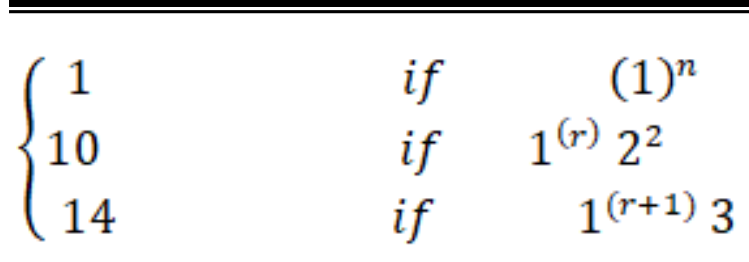

(3.3.11) if $\mu=(r, 1,1,1,1)$, the standard rows in red in the following tables will be according to the cycle length as follows:

$\left\{\begin{array}{ccc}1 & \text { if } & (1)^{n} \\ 21 & \text { if } & 1^{(r)} 2^{2} \\ 24 & \text { if } & 1^{(r+1)} 3\end{array}\right.$

(3.3.12) if $\mu=(r, 2,2,1)$, the standard rows in dark grey in the following tables will be according to the cycle length as follows:
$\left\{\begin{array}{c}1 \\ 15 \\ 17\end{array}\right.$
if
$(1)^{n}$
if $\quad 1^{(r)} 2^{2}$
if $\quad 1^{(r+1)} 3$

(3.3.13) if $\mu=(r, 2,1,1,1)$, the standard rows in dark brown in the following tables will be according to the cycle length as follows:

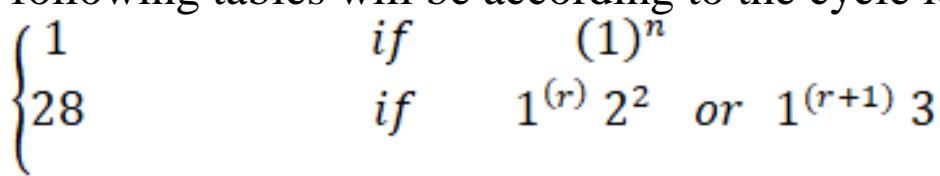

(3.3.14) if $\mu=(r, 1,1,1,1,1)$, the standard rows in pink in the following tables will be according to the cycle length as follows:

$\left\{\begin{array}{ccr}1 & \text { if } & (1)^{n} \\ 55 & \text { if } & 1^{(r+1)} 2^{2} \\ 45 & \text { if } & 1^{(r+2)} 3\end{array}\right.$

Hereafter, the tables $A_{2}$ to $A_{7}$ are sequenced where all the previously mentioned cases are illustrated, each case is given a specific color: 


\begin{tabular}{|l|l|}
\hline $\mathrm{A}_{2}$ & \\
\hline $\begin{array}{l}\text { Cycle type } \\
\text { Partition }\end{array}$ & $1^{2}$ \\
\hline$(2)$ & 1 \\
\hline$(1,1)$ & 1 \\
\hline & $(3.4)$ \\
\hline
\end{tabular}

\begin{tabular}{|l|l|l|}
\hline \multicolumn{2}{|l|}{$\mathrm{A}_{3}$ Cycle type } & \multicolumn{2}{l|}{} \\
\hline partition & $1^{3}$ & 3 \\
\hline$(3)$ & 1 & 0 \\
\hline$(2,1)$ & 1 & 1 \\
\hline$(1,1,1)$ & 1 & 2 \\
\hline
\end{tabular}

(3.5)

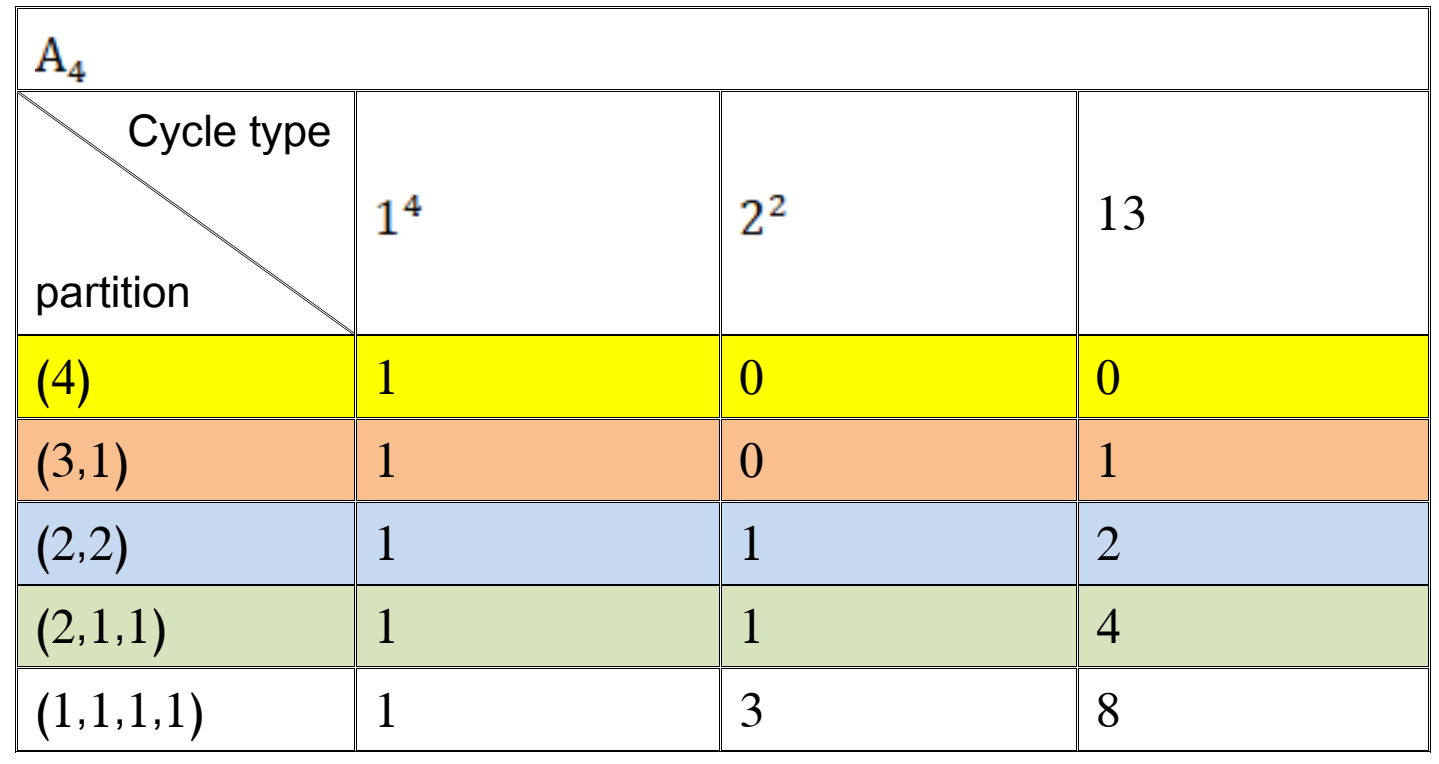

(3.6) 
The Relation Between the Alternating Group and Standard ...

\begin{tabular}{|c|c|c|c|c|}
\hline \multicolumn{5}{|l|}{$\mathrm{A}_{5}$} \\
\hline $\begin{array}{l}\text { Gycle type } \\
\text { partition }\end{array}$ & $1^{5}$ & $12^{2}$ & $1^{2} 3$ & 5 \\
\hline (5) & 1 & 0 & 0 & 0 \\
\hline$(4,1)$ & 1 & 0 & 1 & 1 \\
\hline$(3,2)$ & 1 & 1 & 2 & 2 \\
\hline$(3,1,1)$ & 1 & 1 & 4 & 4 \\
\hline$(2,2,1)$ & 1 & 3 & 6 & 6 \\
\hline$(2,1,1,1)$ & 1 & 6 & 11 & 12 \\
\hline$(1,1,1,1,1)$ & 1 & 15 & 20 & 24 \\
\hline
\end{tabular}

(3.7)

\begin{tabular}{|c|c|c|c|c|c|c|}
\hline \multicolumn{7}{|l|}{$\mathrm{A}_{6}$} \\
\hline partition & $1^{6}$ & $1^{2} 2^{2}$ & $1^{3} 3$ & 24 & $3^{2}$ & 15 \\
\hline (6) & 1 & 0 & 0 & 0 & 0 & 0 \\
\hline$(5,1)$ & 1 & 0 & 1 & 0 & 0 & 1 \\
\hline$(4,2)$ & 1 & 1 & 2 & 1 & 1 & 3 \\
\hline$(4,1,1)$ & 1 & 1 & 4 & 2 & 1 & 6 \\
\hline$(3,2,1)$ & 1 & 3 & 6 & 5 & 3 & 12 \\
\hline$(3,1,1,1)$ & 1 & 6 & 11 & 12 & 6 & 24 \\
\hline$(3,3)$ & 1 & 1 & 2 & 1 & 1 & 4 \\
\hline$(2,2,2)$ & 1 & 6 & 8 & 9 & 6 & 18 \\
\hline$(2,2,1,1)$ & 1 & 10 & 14 & 19 & 10 & 36 \\
\hline$(2,1,1,1 ، 1)$ & 1 & 21 & 24 & 42 & 20 & 72 \\
\hline$(1,1,1,1,1 ، 1)$ & 1 & 45 & 40 & 90 & 40 & 144 \\
\hline
\end{tabular}




\section{Ilham Matta Yacoob, Hadil H. Sami \& Mohammed Kassim Ahmed}

\begin{tabular}{|c|c|c|c|c|c|c|c|c|}
\hline \multicolumn{9}{|l|}{$\mathrm{A}_{7}$} \\
\hline $\begin{array}{l}\text { Cycle type } \\
\text { partition }\end{array}$ & $1^{7}$ & $1^{3} 2^{2}$ & $1^{4} 3$ & $2^{2} 3$ & $3^{2} 1$ & 421 & $51^{2}$ & 7 \\
\hline (7) & 1 & 0 & 0 & 0 & 0 & 0 & 0 & 0 \\
\hline$(6,1)$ & 1 & 0 & 1 & 0 & 0 & 0 & 1 & 1 \\
\hline$(5,2)$ & 1 & 1 & 2 & 0 & 1 & 1 & 3 & 3 \\
\hline$(5,1,1)$ & 1 & 1 & 4 & 0 & 1 & 2 & 6 & 6 \\
\hline$(4,2,1)$ & 1 & 3 & 6 & 2 & 5 & 8 & 14 & 15 \\
\hline$(4,1,1,1)$ & 1 & 6 & 11 & 3 & 9 & 18 & 27 & 30 \\
\hline$(4,3)$ & 1 & 1 & 2 & 1 & 2 & 2 & 5 & 5 \\
\hline$(3,2,2)$ & 1 & 6 & 8 & 6 & 12 & 19 & 26 & 30 \\
\hline$(3,2,1,1)$ & 1 & 10 & 14 & 12 & 22 & 41 & 50 & 60 \\
\hline $1(3,1,1,1$ & 1 & 21 & 24 & 24 & 44 & 90 & 96 & 120 \\
\hline$) 1(3,3$ & 1 & 3 & 6 & 4 & 7 & 11 & 18 & 20 \\
\hline$(2,2,2,1)$ & 1 & 15 & 17 & 21 & 36 & 66 & 72 & 90 \\
\hline$(2,2,1,1,1)$ & 1 & 28 & 28 & 44 & 69 & 142 & 138 & 180 \\
\hline $1,1,1,1), 1,(2$ & 1 & 55 & 45 & 94 & 140 & 301 & 264 & 360 \\
\hline $1,1,1,1), 1,(1,1$ & 1 & 105 & 70 & 210 & 280 & 630 & 504 & 720 \\
\hline
\end{tabular}




\section{$\underline{\text { References }}$}

[1] I.N. Herstein " Topics in Algebra" $2^{\text {nd }}$ edition university of Chicago 1975.

[2] T. W. Judson \& Stephen F. " Abstract algebra theory and applications" Austin state university, 2010

[3] R. Keown “ An introduction to Group Representation Theory “ Academic . press, New York, 1975

[4] M.W. Kirson "Introductory Algebra for Physicists" ch. Irreducible representations, 2013.

[5] A. Mathas "Iwahori - Hecke Algebras and Schur Algebras of the Symmetric Groups", Amer.Math. Soc., University Lecture Series, Vol.15, 1999

[6] W. R. Scott “ Group theory” Dover publications, 1987

[7] Y. Zhao "Young Tableaux and the Representations of the Symmetric Group" Harvard College Mathematics Review 2(2), 3345, 2008. 